\title{
Development of a muon radiographic imaging electronic board system for a stable solar power operation
}

\author{
Tomohisa Uchida ${ }^{1}$, Hiroyuki K. M. Tanaka ${ }^{2}$, and Manobu Tanaka ${ }^{3}$ \\ ${ }^{1}$ University of Tokyo, 7-3-1 Hongo, Bunkyo-ku, Tokyo 113-0033, Japan \\ ${ }^{2}$ Earthquake Research Institute, University of Tokyo, Yayoi, Bunkyo-ku, Tokyo, Japan \\ ${ }^{3}$ High Energy Accelerator Research Organization (KEK), 1-1 Oho, Tsukuba 305-0801, Japan
}

(Received October 29, 2008; Revised March 23, 2009; Accepted March 28, 2009; Online published February 22, 2010)

\begin{abstract}
Cosmic-ray muon radiography is a method that is used to study the internal structure of volcanoes. We have developed a muon radiographic imaging board with a power consumption low enough to be powered by a small solar power system. The imaging board generates an angular distribution of the muons. Used for realtime reading, the method may facilitate the prediction of eruptions. For real-time observations, the Ethernet is employed, and the board works as a web server for a remote operation. The angular distribution can be obtained from a remote PC via a network using a standard web browser. We have collected and analyzed data obtained from a 3-day field study of cosmic-ray muons at a Satsuma-Iwojima volcano. The data provided a clear image of the mountain ridge as a cosmic-ray muon shadow. The measured performance of the system is sufficient for a stand-alone cosmic-ray muon radiography experiment.
\end{abstract}

Key words: Cosmic-ray muon radiography, imaging system.

\section{Introduction}

Recent technological advances in cosmic-ray muon radiography (Nagamine et al., 1996) has enabled scientists to better determine the internal structure of a volcano. Cosmic-ray muons, which are characterized by small horizontal angles at sea level, are high-energy particles that can penetrate through a volcano. The amount of energy lost by muons while passing through matter is dependent on the density of that matter. The resultant angular distribution of the muons can then be used to calculate the density profile of a volcano and, consequently, its internal structure. Therefore, a cosmic-ray muon radiography detection system is needed to determine the paths of these muons and to count the events. Various volcanoes have recently been observed by a detection system using a nuclear emulsion film (Tanaka et al., 2007a, b, c). In terms of space limitations associated with these systems near volcanoes, however, a compact system would be useful. However, we are not able to use this system for real-time observations, and real-time readings may facilitate the predictions of volcanic eruptions. A system for real-time observation requires readout electronics. A number of systems already exist for this purpose (Tanaka et al., 2001, 2003, 2005), but since these systems are designed based on NIM (U.S. NIM Committee, 1990) and CAMAC (IEEE, 1982) system, power consumption is large and the systems require commercial power supplies. It is, therefore, difficult to construct such systems near volcanoes. In order to overcome this difficulty, we have de-

Copyright (c) The Society of Geomagnetism and Earth, Planetary and Space Sciences (SGEPSS); The Seismological Society of Japan; The Volcanological Society of Japan; The Geodetic Society of Japan; The Japanese Society for Planetary Sciences; TERRAPUB.

doi:10.5047/eps.2009.03.002 veloped a power-effective readout board with a power consumption that can be fed by a small solar power system.

\section{Detection System}

Here we briefly discuss a detection system for cosmicray muon radiography before going in a detailed discussion of the readout board.

The detection system detects cosmic-ray muons penetrating through a volcano, determines the paths of these muons, and generates the angular distribution of the muons as a histogram.

A block diagram of the detection system is shown in Fig. 1. The system consists of two major parts-a detector and a readout part. The detector part detects the cosmic-ray muons using two position-sensitive counters. The readout system processes signals from the counters and generates a histogram for an angular distribution.

Figure 2 shows a schematic view of the counter, which consists of two planes of plastic-scintillator arrays. The $X$ plane and $Y$-plane detect the horizontal and vertical crossing position of a muon, respectively. One plane consists of 12 scintillators of $15 \times 70 \times 1000 \mathrm{~mm}$ in size. A photomultiplier is mounted on each scintillator. In total, there are 48 electronic channels for the two counters.

All signals of the photomultipliers are processed by the readout system, which consists of a readout board, Ethernet bridges, and a personal computer (PC). The readout board processes the signals from the detector, generates a histogram as a web page, and works as a web server. The detector and readout part are connected through a computer network with the Ethernet bridges. The generated histogram can be obtained from the remote PC using a web browser. This board plays a central role in this system 


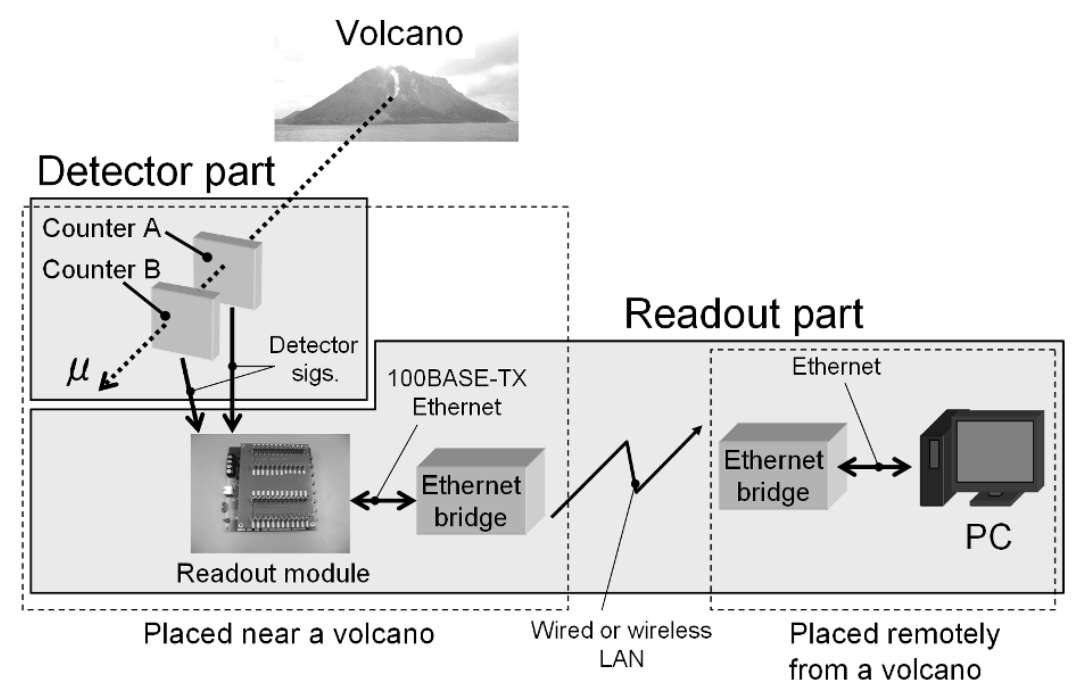

Fig. 1. Detection system for cosmic-ray muon radiography.

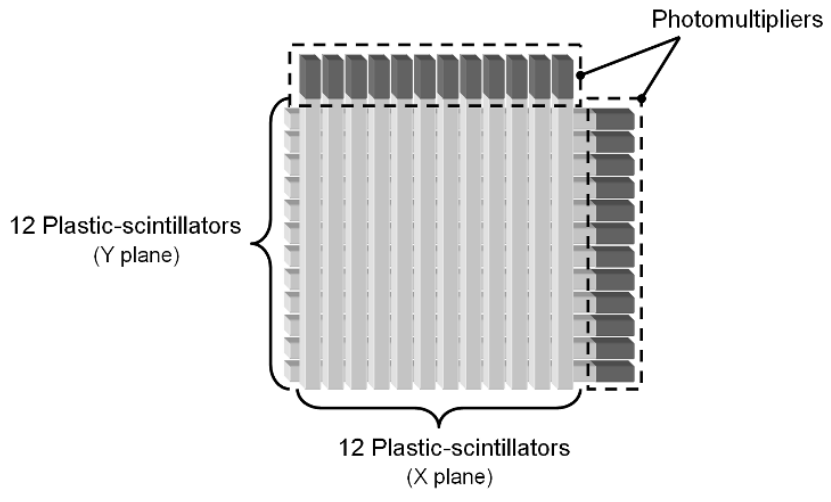

Fig. 2. Schematics of the scintillating counter.

(see following section). In addition, all devices, except for the readout board, are commercial products, and there are many commercial products at reduced price which can be used for this application. Thus, a cost-effective and highperformance system can be easily constructed.

For communication, a wired or wireless local area network (LAN) can be employed. In cases in which a small solar power system and wireless bridges are used, it is possible to construct a remote observation station near a volcano. This is a major advantage of this system. The low power consumption requirement allows the utilization of a small solar power system-a very important factor in the development of this system. For an observation system near a volcano, the system requires a power supply capability of about $600 \mathrm{~W}$. When the sunlight is taken into account, an effective average power of about $60 \mathrm{~W}$ can be obtained. Another important factor to be considered in the development of this system is the fact that near a volcano access could be limited, thereby constraining the size of the station. The construction costs of the station can be reduced by keeping the station small and using light-weight elements. In order to resolve these issues, we have developed a specific readout board. For this system, the goal was to have a reduced power consumption board (several watts) compared to the effective available average power. The second goal was to have a single module of reduced size and weight (several hundreds grams).

\section{Readout Board}

The readout board is discussed in detail in this section. As already mentioned, the board processes the incoming signals from detectors and generates a histogram. The PC can read the histogram from the readout board with a web browser.

This board consists of a main and a daughter board, which is $35 \times 160 \times 160 \mathrm{~mm}$ in size and weighs $420 \mathrm{~g}$. Figure 3 shows photographs of these boards. The daughter board, mounted on the main board, is a connector adapter so as to allow the use of various types of connectors. The signals are processed by the main board, and the main parts of this board are comparators, a field programmable gate array (FPGA, Xilinx Spartan3AN-700; XILINX, 2007), and an Ethernet physical layer device (PHY, SMSC LAN8700i; SMSC, 2007).

Figure 4 shows a block diagram of the readout board that consists of NIM connectors, comparators, an event filter, a histogram generator, a network processor, and an Ethernet PHY. The FPGA works on a $50-\mathrm{MHz}$ system clock. The event filter, the histogram generator, and the network processor are implemented on the single FPGA.

The detector signals are received by the comparators and are digitized after they have been compared with threshold voltages. The event filter selects candidate muon events for which the muon path can be constructed and generates information data on the paths. The data consist of a detection time and detection positions of the two counters. A histogram that depicts the angular distribution of the selected events is generated by the histogram generator using the path information. The network processor handles network protocols (Stevens, 1994) to access the histogram from a remote PC using a web browser. The network protocols used for this purpose are Ethernet, Internet Protocol (IP), Trans- 


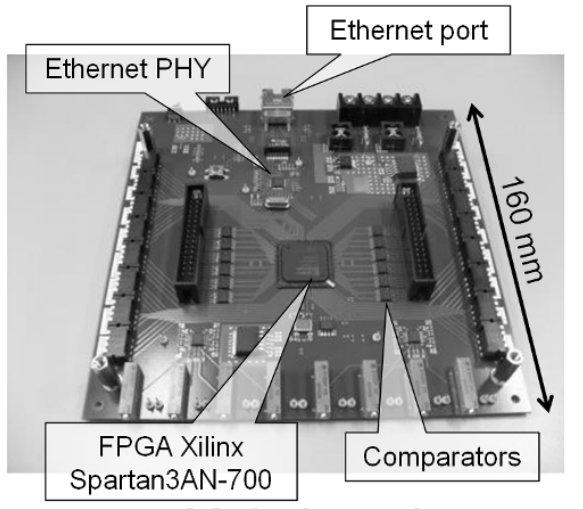

Main board

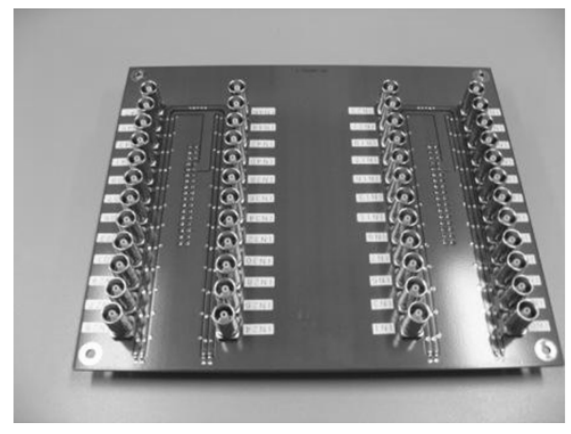

Daughter board

Fig. 3. Photographs of a main and daughter board.

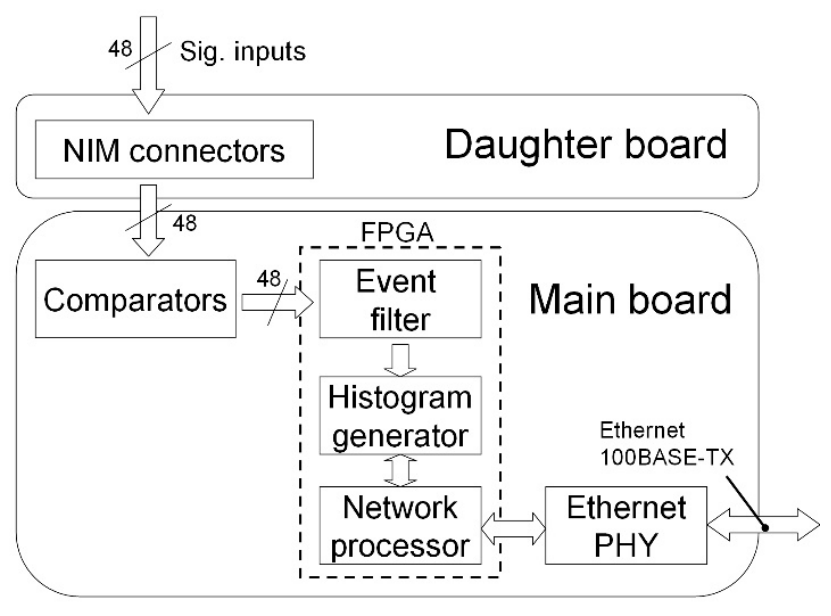

Fig. 4. Block diagram of the readout board.

mission Control Protocol (TCP), Hyper Text Transfer Protocol (HTTP), and Hyper Text Markup Language (HTML). The Ethernet PHY converts signals to meet Ethernet specifications. The FPGA circuits are discussing in more detail in the following sections.

\subsection{Event filter}

The event filter selects events that can be used to construct the muon paths and generates path information.

Figure 5 shows a block diagram of the event filter which consists of samplers and a coincidence unit. Input signals from the comparators are sampled at $200 \mathrm{MHz}$ (period $5 \mathrm{~ns}$ ). The sampled signal is stretched to $40 \mathrm{~ns}$ when the pulse width is shorter than $40 \mathrm{~ns}$ in order to be in synchronization with the system clock. The coincidence unit selects events from the sampled signals and generates the information on the candidate muon path. The selection criteria are as follows: there is one signal from each scintillator-array plane, the two signals of the counter are detected simultaneously, and the four signals are in a coincidence window. The window size can be set from 0 to $2 \mathrm{~s}$ in steps of $20 \mathrm{~ns}$. An angle of the incident muon is calculated using the difference between detection positions of two counters. The path information consists of two 5-bit-width data sets containing

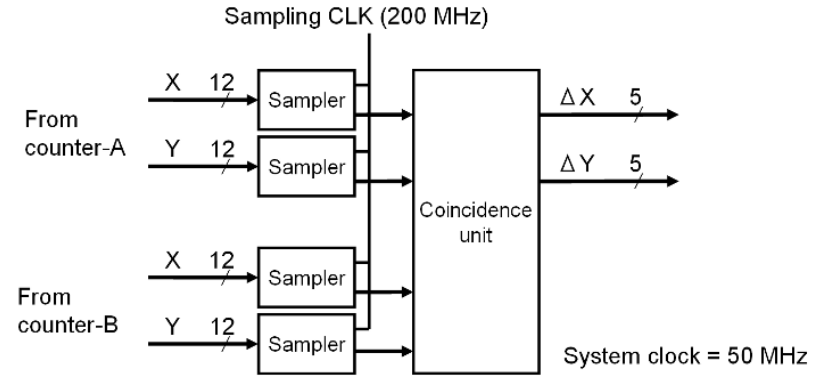

Fig. 5. Block diagram of the event filter.

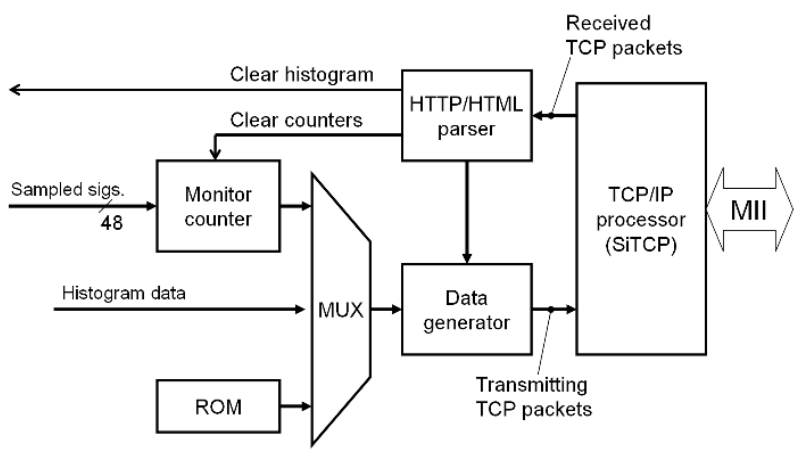

Fig. 6. Block diagram of the network processor.

the detection position differences for horizontal and vertical scintillating strips, respectively.

\subsection{Histogram generator}

The histogram is generated in a 32-bit-width internal memory of the FPGA. The path information, 10 bit in width and generated by the coincidence unit, is used as the address for access to the internal memory. The data according to the address are counted up when an event is detected. These data are read by the network processor described in the next section, using a remote PC.

\subsection{Network processor}

The network processor handles the network protocols used to access the histogram from a remote PC using a web browser. The PC can execute instructions as follows: obtain and clear histogram data, and obtain and clear data of event 


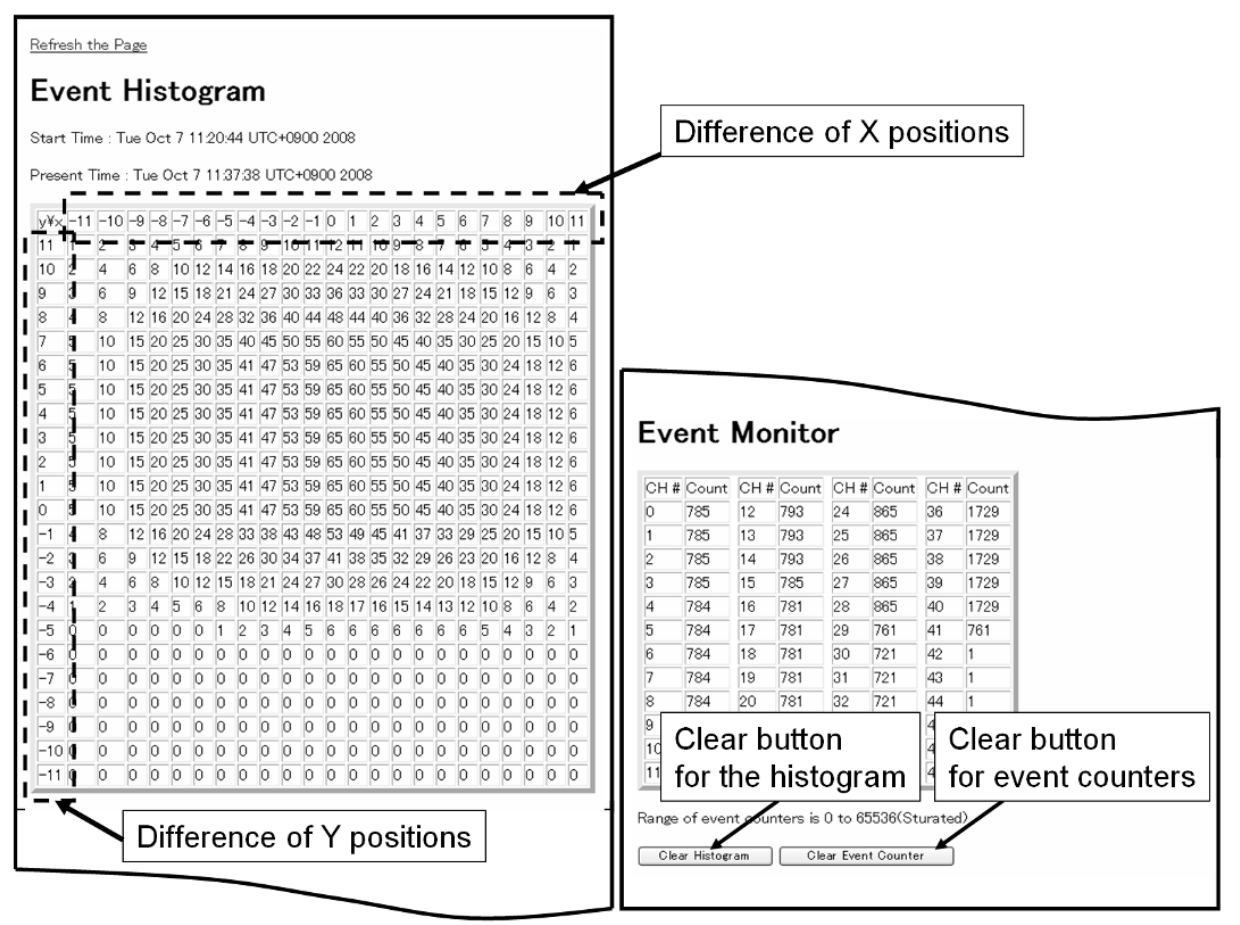

Fig. 7. Web page of the histogram.

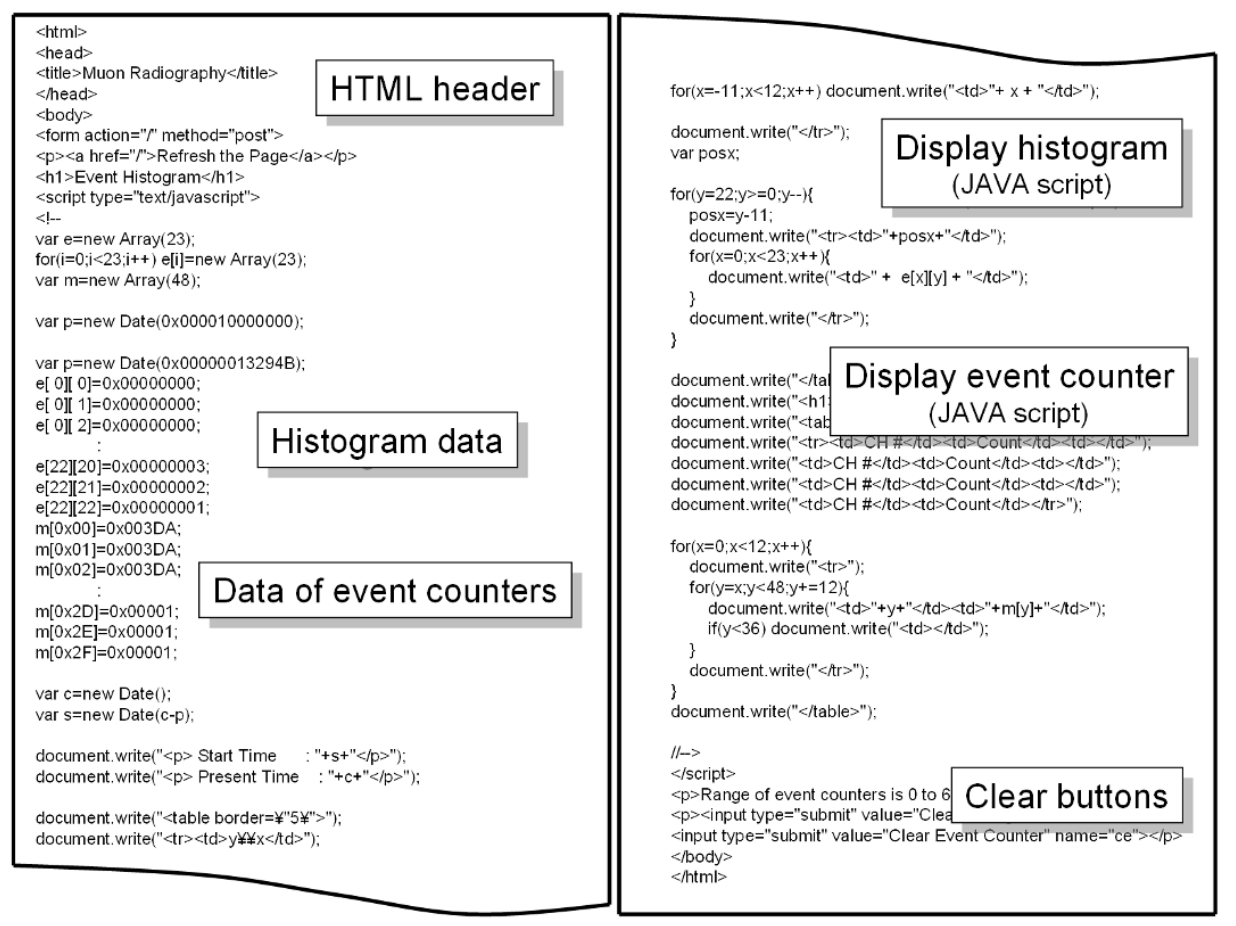

Fig. 8. HTML source file for the web page.

counters for monitor of the detectors.

The feature of this block is that those network protocols are processed by only a hardware circuit specialized for a web page. There is no CPU and no other programmable sequencers in this block. This design has advantages in terms of power consumption and operating failure rate. The protocols are generally processed by a system employing an embedded CPU with an operating system (OS). The system requires an external memory device because the size of the internal memories is too small to store a program. The use of a small number of devices contributes to a reduced power consumption and failure rate while running.

Figure 6 shows a block diagram of the network processor, which consists of a TCP/IP processor (SiTCP) (Uchida and Tanaka, 2006; Uchida, 2008), a HTTP/HTML parser, event counters, a read only memory (ROM), a multiplexer 
(MUX), and a data generator. This board works as a web server. The network protocols processed by this block are HTML, HTTP, TCP, IP, and Ethernet. The SiTCP, which is a hardware-based TCP/IP processor that can be implemented on a FPGA with no other external devices (such as memory devices; Uchida and Tanaka, 2006; Uchida, 2008), processes TCP, IP, and Ethernet. The parser analyzes HTML and HTTP data extracted from the TCP packets by the SiTCP and executes commands from the remote PC. There are three commands-clear histogram data; clear event counters; obtain histogram data. There are distinct buttons for the histogram data and event counters on a web page (see Fig. 7). A command packet to clear histogram data or event counters is transmitted from the PC when the clear button is clicked. Acknowledge packets for the commands are replied to by this block when the histogram data or the event counters are cleared. To measure the counting rate of the detectors, the event counters total the detector signals for $1 \mathrm{~s}$ after the clear command is received.

Figure 8 shows a HTML source file of the web page, which consists of three parts - fixed data; histogram data; values of event counters. The fixed data are a HTML header, HTML codes, and Java scripts. The Java scripts are used to reduce the load of this board. The fixed data are read from the ROM, and the histogram data and values of event counters are read from the histogram memory and event counters, respectively. These data are selected by the data generator with the MUX when data for the web page are generated. The selected data are forwarded to the SiTCP and transmitted to a PC. The histogram is displayed as a web page on the remote PC finally.

\section{Tests and Results}

We measured the power consumption of the readout board and observed a volcano with this system. The first image was obtained as a density profile.

A measured power consumption of the readout board is $2.5 \mathrm{~W}$. This result shows that a small solar power system is enough for this application.

Using this board, we observed the Mt. Iwodake volcano

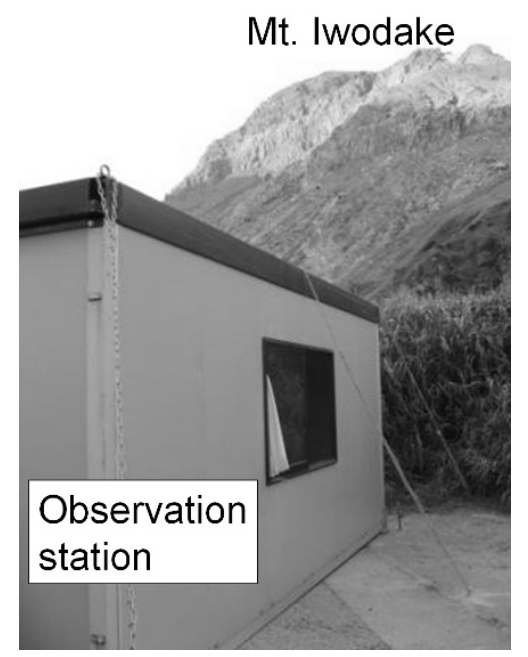

Fig. 9. Photograph of the observation station and Mt. Iwodake.



Fig. 10. Photograph of the detection system installed in the station.

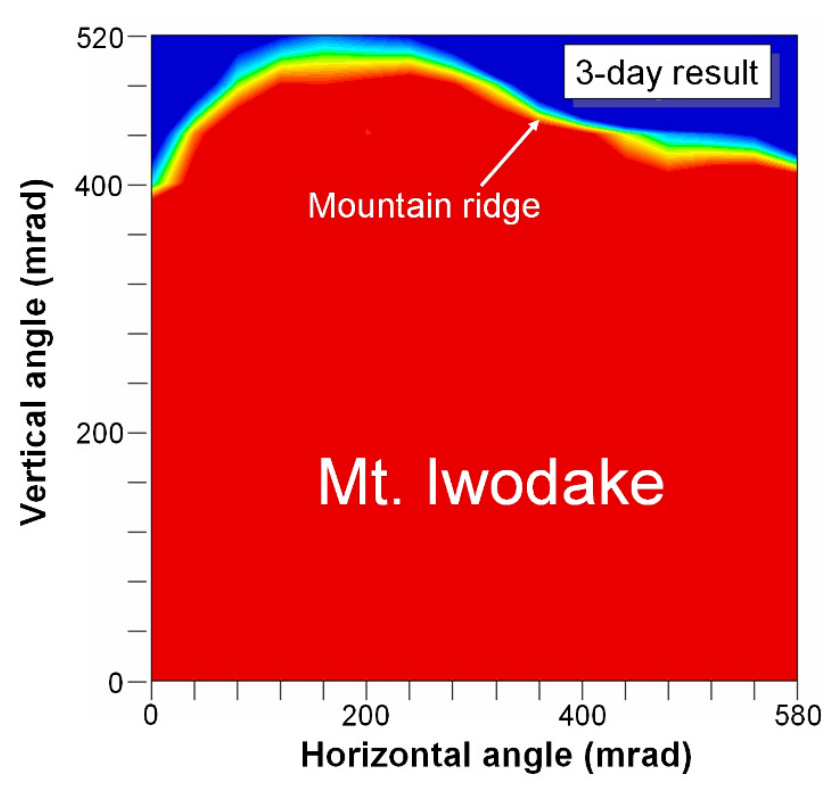

Fig. 11. A radiographic image of Mt. Iwodake. The red and blue areas represent high and low densities, respectively.

in July 2008, whose main peak is $703 \mathrm{~m}$ a.s.l. This volcano is located on Iwojima Island, in the southwest region of Japan (about $1000 \mathrm{~km}$ from Tokyo). We constructed a small observation station with a prefabricated house near Mt. Iwodake at a distance of about $1.2 \mathrm{~km}$ from the main peak. A photograph of the observation station is shown in Fig. 9. The mountain behind the station is Mt. Iwodake. Figure 10 shows the detection system used for this observation. One of the two counters and the readout board are shown. Since commercial power is supplied to this station, we did not employ a solar power system for this observation.

Figure 11 shows the first result. This is a radiographic image of Mt. Iwodake showing a density profile integrated along the paths of the muons. The red area represents a relatively more dense material (relatively low counting rate of detected muons). The blue area represents a relatively 
low dense material (relatively high counting rate of detected muons). This result was obtained during 3 days of data collecting. The result image has been obtained by the following process: correcting efficiencies of detectors; removing high-frequency components; normalizing by the maximum value (i.e., the blue area). The sky and the volcano appear distinctly as blue and red areas, respectively. We can clearly recognize the line of the mountain ridge. This result shows that performance of the readout board is sufficient for cosmic-ray muon radiography experiments because the relative densities can be measured.

\section{Summary}

We have developed a muon radiographic imaging board for use in a stable solar power system. The board has a low power consumption, $2.5 \mathrm{~W}$, and a small size, $35 \times 160 \times 160 \mathrm{~mm}$, and weighs only $420 \mathrm{~g}$. All detector signals are processed by a single board. A histogram of the angular distribution of cosmic-ray muons is generated on the board. A remote PC can communicate with the board and obtain the histogram through LAN using a web browser. This board enables us to employ a small solar power system and a wireless LAN system and consequently, to install a detection system near a volcano where there is no commercial power and limited access to an observation station. Using this system, we have been able to collect and analyze data from a 3-day-long cosmic-ray muon observation at Satsuma-Iwojima volcano. A clear image of the mountain ridge was obtained as a cosmic-ray muon shadow. This result shows that the performance of the system is sufficient for the experiments. We are now ready use test the cosmicray muon radiography system on various other volcanoes.

\section{References}

IEEE, Standard Modular Instrumentation and Digital Interface System (CAMAC), IEEE-583, January, 1982
Nagamine, K., M. Iwasaki, K. Shimomura, and K. Ishida, Method of probing inner-structure of geophysical substance with the horizontal cosmicray muons and possible application to volcanic eruption prediction, Nucl. Instr. Meth. A, 356, 585-595, 1996.

SMSC, LAN8700/LAN8700i data sheet, Rev. 1.5, October, 2007

Stevens, W. R., TCP/IP Illustrated, Volume 1: The protocols, AddisonWesley, 1994.

Tanaka, H., K. Nagamine, N. Kawamura, S. Nakamura, K. Ishida, and K. Shimomura, Development of the Cosmic-Ray Muon Detection System for Probing Internal-Structure of a Volcano, Hyperfine Inter., 138, 521526, 2001.

Tanaka, H., K. Nagamine, N. Kawamura, S. Nakamura, K. Ishida, and K. Shimomura, Development of a two-fold segmented detection system for near horizontally cosmic-ray muons to probe the internal structure of a volcano, Nucl. Instr. Meth. A, 507, 657-669, 2003.

Tanaka, H., K. Nagamine, N. Nakamura, and K. Ishida, Radiographic measurements of the internal structure of Mt. West Iwate with nearhorizontal cosmic-ray muons and future developments, Nucl. Instr. Meth. A, 555, 164-172, 2005.

Tanaka, H., T. Nakano, S. Takahashi, J. Yoshida, and K. Niwa, Development of an emulsion imaging system for cosmic-ray muon radiography to explore the internal structure of a volcano, Mt. Asama, Nucl. Instr. Methods A, 575, 489-497, 2007a.

Tanaka, H., T. Nakano, S. Takahashi, J. Yoshida, M. Takeo, J. Oikawa, T. Ohminato, Y. Aoki, E. Koyama, H. Tsuji, and K. Niwa, High resolution imaging in the inhomogeneous crust with cosmic-ray muon radiography: The density structure below the volcanic crater floor of Mt. Asama, Japan, Earth Planet. Sci. Lett., 263, 104-113, 2007 b.

Tanaka, H., T. Nakano, S. Takahashi, J. Yoshida, H. Ohshima, T. Maekawa, H. Watanabe, and K. Niwa, Imaging the conduit shape beneath the dome with cosmic-ray muons: the structure beneath Showa-Shinzan lava dome, Japan, Geophys. Res. Lett., 34, L22311, 2007c.

Uchida, T., Hardware-based TCP processor for gigabit ethernet, IEEE Tran. Nucl. Sci., 55, 1631-1637, 2008.

Uchida, T. and M. Tanaka, Development of TCP/IP processing hardware, IEEE Nuclear Science Symposium 2006 Conference Record, 14111414, 2006.

U.S. NIM committee, Standard NIM Instrumentation System, DOE/ER0457T, May, 1990.

XILINX Inc., Spartan-3AN FPGA Family: Data Sheet, DS557, December, 2007.

T. Uchida (e-mail: uchida@post.kek.jp), H. K. M. Tanaka, and M. Tanaka 